\title{
SEVERE INTERSTITIAL LUNG DISEASE AS FIRST PRESENTATION OF ANTISYNTHETASE SYNDROME: CASE REPORT
}

Milene Tieno Sakata Vasconcellos ${ }^{1, \star}$, Giovana Rosa Gameiro ${ }^{1}$, Rodrigo Maldonado Franco ${ }^{1}$, Gustavo Lawim ${ }^{1}$, Bruno Carnevalli¹,

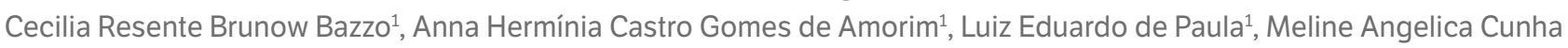
Rotter Ferreira ${ }^{1}$, Neide Tomimura Costa ${ }^{1}$

1.Universidade Estadual de Londrina, Londrina (PR), Brazil.

*Corresponding author: milenetieno@gmail.com

\section{BACKGROUND}

Antisynthetase syndrome (AS) is a rare systemic autoimmune disease considered part of the inflammatory myopathies' subgroup. It is characterized by antibodies against aminoacyl tRNA synthetase, especially anti-Jo-1, along with clinical features that can include myositis, arthropathy, fever, mechanic's hands, Raynaud's phenomenon and interstitial lung disease, the latter being the main prognostic indicator.

\section{CASE REPORT}

The patient was a 33-year-old woman, brown, with no significant past medical history, admitted with a 2-month history of progressive dyspnea on exertion, which had worsened 2 days before admission, associated with unmeasured fever and unintentional weight loss. Before admission to our service, she had been previously treated for bacterial pneumonia with the use of antibiotics and home oxygen therapy. Physical examination revealed tachypnea, but no signs of respiratory discomfort, satisfactory oxygen saturation using a nasal cannula at a flow rate of $4 \mathrm{~L} / \mathrm{min}$. Patient was afebrile, with no muscular, cutaneous or articular manifestations. Chest computed tomography (CT) was compatible with nonspecific interstitial pneumopathy (Figure 1) and pulmonary artery ectasia. Testing revealed creatine phosphokinase (CPK) 704 $\mathrm{IU} / \mathrm{L}$, lactate dehydrogenase (LDH) $408 \mathrm{IU} / \mathrm{L}$, aspartate aminotransferase (AST) $71 \mathrm{IU} / \mathrm{L}$, alanine aminotransferase (ALT) 72 IU/L. We proceed with the investigation: anti-Ro positive: $91.8 \mathrm{IU} / \mathrm{mL}$, ANA 1:80 cytoplasmic dotted pattern, and anti-Jo-1 positive: $35.6 \mathrm{IU} / \mathrm{mL}$. Sjögren's syndrome was ruled out as salivary gland biopsy was normal and the patient did not present sicca symptoms. Lung biopsy showed: nonspecific interstitial pneumonia (NSIP), organizing pneumonia, occasional cellular bronchiolitis and peribronchiolar alveolar metaplasia, which suggested either AS or hypersensitivity pneumonitis. Anti-Jo-1 positivity in conjunction with ILD and suggestive biopsy results lead to a presumptive diagnosis of AS. Pulse therapy with methylprednisolone and posteriorly with cyclophosphamide was performed. The patient had clinical improvement and was discharged, but still required home oxygen therapy. To date, the patient is in outpatient follow-up at our rheumatology service, maintaining the use of corticosteroids and monthly cyclophosphamide infusions.

\section{CONCLUSION}

Antisynthetase syndrome is a rare autoimmune condition that can pose a diagnostic challenge. We sought to present a case report of a patient previously healthy, initially presented with only pulmonary manifestations, posteriorly requiring home oxygen therapy, which highly impacted on her quality of life. We emphasize the importance of a thorough medical evaluation and early treatment in order to minimize clinical repercussions and target organ damage.

\section{KEYWORDS}

Antisynthetase syndrome, Interstitial lung disease, Anti-Jo-1, Inflammatory myopathies. 


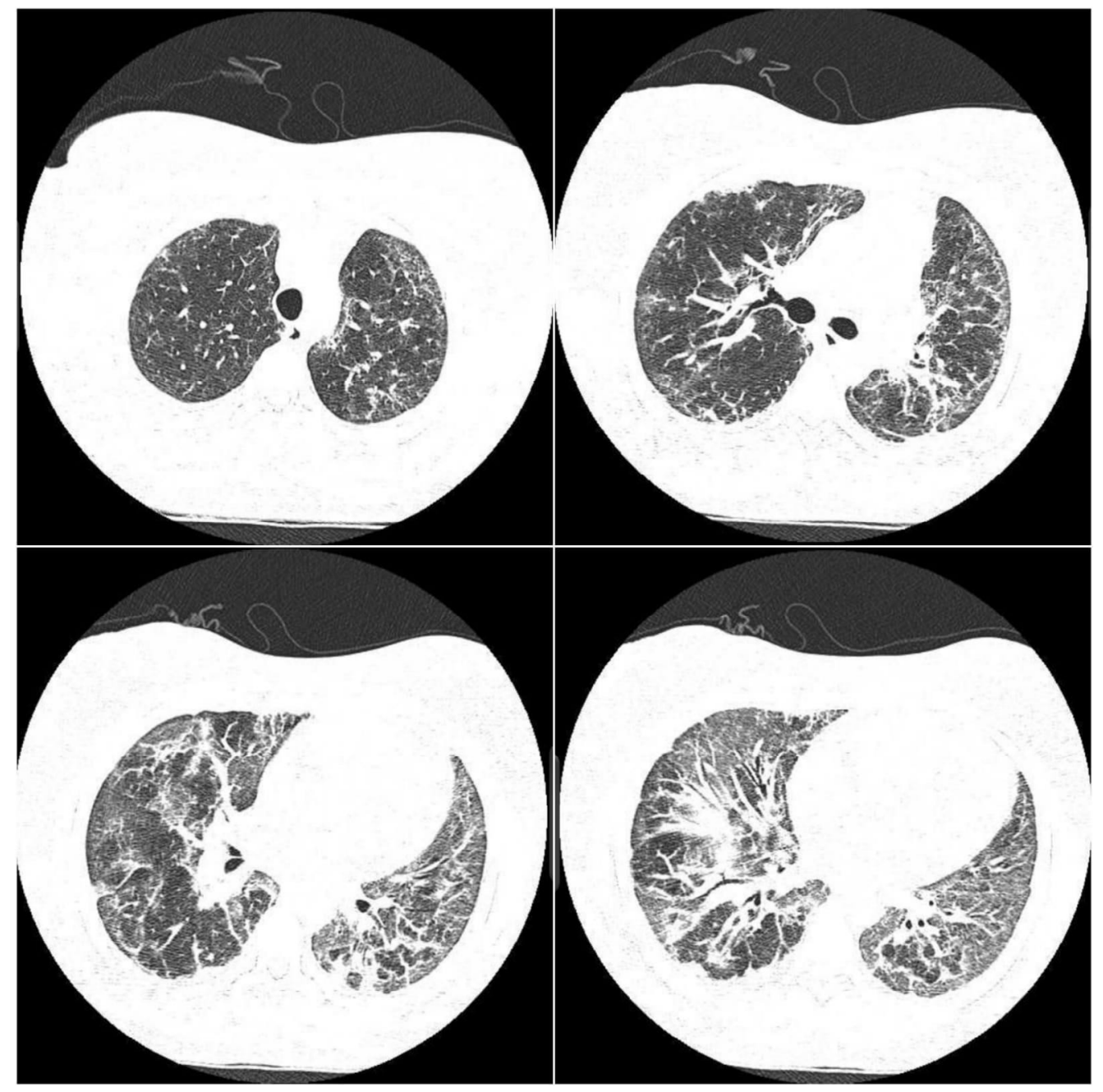

Figure 1. Chest CT: ground-glass opacities associated with septal thickening. 